\title{
Polyarthritis, Tenosynovitis and Dry Eyes after Treatment by Immune Check-Point Inhibitors
}

\author{
Baïdy Sy Kane ${ }^{1,2 *}$, Ahmed Ould-Hennia ${ }^{2}$, Nesrine Karaa-Zbidi', Souhaibou Ndongo ${ }^{1}$, \\ Abdoulaye Pouye ${ }^{1}$, Richard Damade ${ }^{2}$ \\ ${ }^{1}$ Department of Internal Medicine, Le Dantec Teaching Hospital, Cheikh Anta DIOP University, Dakar, Senegal \\ ${ }^{2}$ Department of Rheumatology and Internal Medicine, Chartres Hospital, Chartres, France \\ Email: *baidysy.kane@ucad.edu.sn
}

How to cite this paper: Kane, B.S., OuldHennia, A., Karaa-Zbidi, N., Ndongo, S., Pouye, A. and Damade, R. (2020) Polyarthritis, Tenosynovitis and Dry Eyes after Treatment by Immune Check-Point Inhibitors. Open Journal of Rheumatology and Autoimmune Diseases, 10, 8-13.

https://doi.org/10.4236/ojra.2020.101002

Received: October 24, 2019

Accepted: December 8, 2019

Published: December 11, 2019

Copyright $\odot 2020$ by author(s) and Scientific Research Publishing Inc. This work is licensed under the Creative Commons Attribution International License (CC BY 4.0).

http://creativecommons.org/licenses/by/4.0/

\begin{abstract}
Introduction: Immune checkpoint inhibitors targeting programmed death protein-1 and cytotoxic-T-lymphocyte-antigen-4 have revolutionized the treatment of various cancers. Despite their effectiveness, these therapies can lead to immune related adverse events. Observation: We reported a case of a 43year-old white woman who was referred to our department for a management of acute polyarthritis. She was followed for a relapsing metastatic melanoma (stage IIIb) by surgery and Pembrolizumab, an immune checkpoint inhibitor targeting programmed death protein-1. After receiving her $4^{\text {th }}$ cycle of this therapy she developed arthritis of the knees and the ankles, tenosynovitis and dry eyes with keratitis. After exclusion of other causes of polyarthritis such as connective-tissue disease, the diagnosis of rheumatologic immunerelated adverse events was retained. She was treated by $20 \mathrm{mg}$ of prednisone daily, Pembrolizumab was discontinued. The evolution was favorable. Conclusion: Rheumatologic manifestations secondary to immune checkpoint inhibitors have been less well described in the literature. Their management requires the collaboration of oncologists and rheumatologists to limit the diagnostic delay and for an appropriate therapeutic choice according to their severity.
\end{abstract}

\section{Keywords}

Drug-Related Side Effects and Adverse Events, Rheumatic Immune-Related Adverse Events, Immune Checkpoint Inhibitors, Pembrolizumab, Arthritis

\section{Introduction}

Immune checkpoint inhibitors (ICIs) targeting programmed death protein-1 (PD-1) and cytotoxic-T-lymphocyte-antigen-4 (CTLA-4) have revolutionized the treat- 
ment of various cancers [1] [2]. Indeed, the widespread use of ICIs has led to remarkable clinical outcomes, with complete remissions and sustained clinical responses seen in some patients with previously refractory cancers such as metastatic melanoma, lung cancer, renal cell carcinoma, and Hodgkin lymphoma [3] [4].

However, activating immune system to fight cancer can also lead to immune related adverse events (irAEs) [3] [5].

These irAEs typically involve the skin, intestines, and endocrine system but virtually all organs can be affected [6]. While colitis, hepatitis, pneumonitis and other IRAEs are well documented, irAEs with rheumatologic and musculoskeletal phenotypes are less well described [7] [8].

We reported a case of polyarthritis that developed after treatment with Pembrolizumab, an ICI targeting PD-1.

\section{Observation}

A 43-year-old white woman was referred to our department for a management of acute polyarthritis. She was followed for a superficial spreading melanoma (SSM) of the right leg diagnosed in 2009 and managed by surgery and skin grafting. In May 2018, she had a relapse of his cancer with metastatic lymph node localization of stage IIIb malignant melanoma. Adenectomy and lymph node cleaning were performed and a therapy with ICIby Pembrolizumab has been started. Three days after receiving her 4th cycle of Pembrolizumab, she developed pain and swelling in her knees and ankles. His other complaints were severe physical asthenia and anorexia and dry eyes. There was no fever and she had no history of inflammatory rheumatism.

The physical examination revealed polyarthritis with swelling, warmth and painful limitation of both knees and ankles. Examination of the other joints was normal. Ophthalmological assessment showed keratitis.

Laboratory evaluation was summarized in Table 1.

Plain radiographs of the hands and the feet were normal. Musculoskeletal ultrasound showed effusion in the suprapatellar recess of the knees and in the tibiotalar joints (Figure 1) with a tenosynovitis in the lateral compartment tendons (peroneal tendons).

We performed the puncture of the left tibiotalar joint, under ultrasound-guidance with evacuation of $5 \mathrm{ml}$ of yellow fluid. The white blood cell count was $10^{5} / \mathrm{mm}^{3}$ without bacteria and microcrystal.

Pembrolizumab was discontinued by her oncologist and she was treated by 20 mg of prednisone daily with a good clinical improvement in arthritis.

\section{Discussion}

ICIs that block CTLA-4, PD1 or PD-L1 are expanding treatment options and increasing survival for patients in selected advanced cancers. Thus, multiples agents targeting CTLA-4 (Ipilimumab), PD1 (Pembrolizumab, Nivolumab) or his ligand 
Table 1. Laboratory investigations.

\begin{tabular}{|c|c|}
\hline Laboratory evaluation & Results \\
\hline Blood cells count & $\begin{array}{c}\text { WBCs: } 11.5 \mathrm{G} / 1 \\
\text { with Neutrophils at } 8.7 \mathrm{G} / \mathrm{l} \\
\text { lymphocytes at } 1.4 \mathrm{G} / 1 \\
\text { Hemoglobin at } 9.6 \mathrm{~g} / \mathrm{dl} \\
\text { Platelets at } 759 \mathrm{G} / 1\end{array}$ \\
\hline CRP & $347 \mathrm{mg} / \mathrm{l}$ \\
\hline Blood protein electrophoresis & Gamma globulinemia at $18.7 \mathrm{~g} / \mathrm{l}$ \\
\hline AST & $23 \mathrm{UI} / 1$ \\
\hline ALT & $40 \mathrm{UI} / 1$ \\
\hline Serum Creatinine & $5.6 \mathrm{mg} / \mathrm{l}$ \\
\hline $\mathrm{HBs}$ antigen, anti-HBc & Negatives \\
\hline \multicolumn{2}{|l|}{ Anti-HCV, anti-HIV 1 and 2} \\
\hline Rheumatoid factor & $16 \mathrm{IU} / \mathrm{l}(0-14)$ \\
\hline Antinuclear antibodies & $1 / 80(\mathrm{~N}<1 / 160)$ \\
\hline $\begin{array}{l}\text { Other autoantibodies (anti-CCP, anti-SSA, } \\
\text { anti-SSB, anti-U1-RNP, anti-Sm) }\end{array}$ & Negative \\
\hline
\end{tabular}

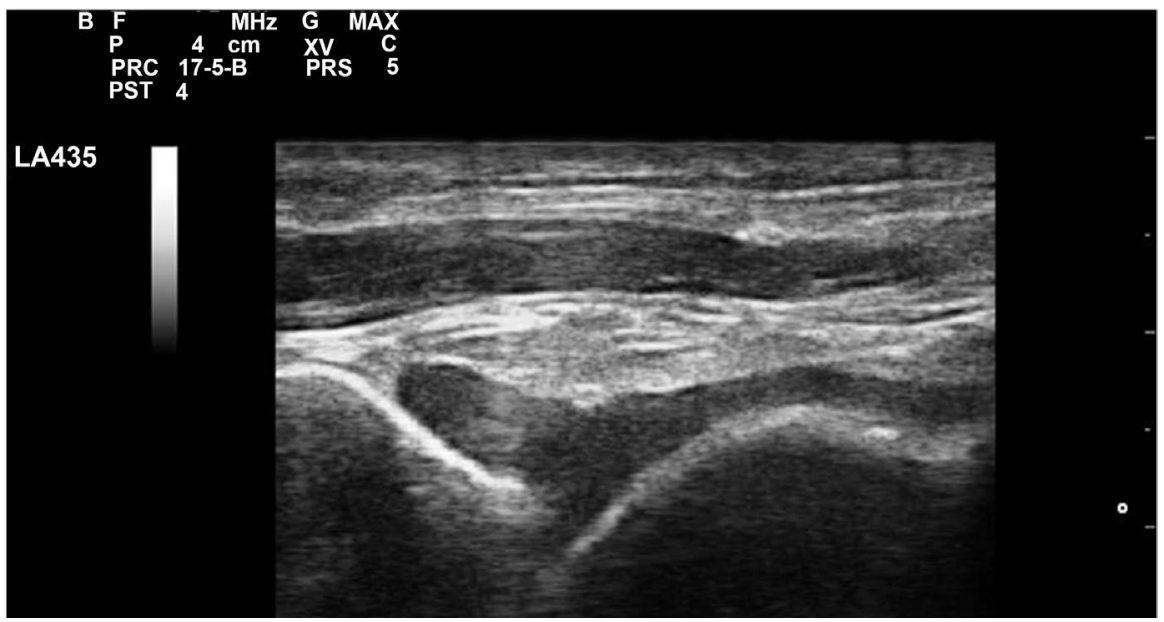

Figure 1. Musculoskeletal ultrasound. Longitudinal scan. Effusion of the Left tibiotalar joint.

PD-L1 (Atezolizumab) have been approved for a variety of indications including metastatic melanoma, non-small-cell lung cancer, renal cell carcinoma, Hodgkin's lymphoma, head and neck cancers and urothelial carcinoma [9] [10].

The adverse events secondary to these molecules are related to their mechanisms of action [11]. Indeed, in the absence of cancer, both CTLA-4 and PD1 play a regulatory role of the interaction between T-cells and self, versus non-self-antigens [10]. Therapeutic blockade of these pathways results in a disruption of the balance between tolerance and immunity. This disruption leads to clinical manifestations widely called irAEs in the literature, which are toxicities that are autoimmune or autoinflammatory in origin [9]. Other authors have proposed the terminology of opportunistic autoimmunity secondary to cancer immunotherapy (OASI) [12]. 
These irAREs occur more frequently with anti-CTLA 4 than anti-PD1 [9] [10]. We reported a case of polyarthritis, tenosynovitis and sicca syndrome induced by Pembrolizumab. This presentation is unusual. Diarrhea, asthenia, itching and rash are the most common irAEs associated with Pembrolizumab in the literature [6]. Rheumatologic irAEs (Rh-irAEs) had been reported with a wide spectrum of clinical manifestations including arthralgias, myalgias, inflammatory arthritis, rheumatoid arthritis-like, polymyalgia rheumatica-like, lupus-like sicca syndrome, myositis and vasculitis [3] [13]. We described a case of polyarthritis that were reported only on five clinical trials with a range of $1 \%-7 \%$ in the meta-analysis of Capelli et al. Dry eyes were most commonly reported with a range of incidence of $3 \%$ $24 \%$ In this study, two cases of polyarthritis and tenosynovitis after treatment of Pembrolizumabsimilar to our observation, have been also reported [7]. Time to onset of polyarthritis is generally $4-8$ weeks after initiation of ICIs, however they have been reported after one dose or up two years after receiving these therapies [3] [14]. In the majority of cases, the auto-antibodies are negative, although Rheumatoid Factor (RF) or anti-citrullinated cyclic peptide positive cases have been reported [3] [15]. In our observation RF was positive with a low titer; other auto-antibodies were negative.

Rh-irAES are managed according to severity assessed using Common Terminology Criteria for Adverse Events grading system [10]. Nonsteroidal anti-inflammatory drugs, Corticosteroids, conventional synthetic anti-rheumatic drugs (e.g. methotrexate, sulfasalazine, leflunomide) and anti-cytokine therapies (e.g. Infliximab or Tocilizumab) can be used in the management of Rh-IRAEs [1]. Temporary discontinuation of Pembrolizumab and corticosteroid were effective in our patient. The main limitation of our work is its nature which not allow draw definitive conclusion. However, this case report should contribute to a better knowledge of Rh-irAEs.

\section{Conclusions}

ICIs have revolutionized the cancer therapy. Despite their effectiveness, ICIs can cause IRAEs such as Rh-irAEs.

Polyarthritis with tenosynovitis and dry eyes are rare complications of Pembrolizumab.

They require the collaboration of oncologists and rheumatologists or internists to limit diagnostic delay and for an appropriate therapeutic choice.

The management is according to their severity. Corticosteroid remains the mainstay therapy for acute irAEs.

\section{Conflicts of Interest}

The authors declare that they have no competing interests.

\section{Consent for Publication}

Written informed consent for publication was obtained from the patients. 


\section{References}

[1] Kim, S.T., Tayar, J., Trinh, V.A., Suarez-Almazor, M., Garcia, S., Hwu, P., Johnson, D.H., Uemura, M. and Diab, A. (2017) Successful Treatment of Arthritis Induced by Checkpoint Inhibitors with Tocilizumab: A Case Series. Annals of the Rheumatic Diseases, 76, 2061-2064. https://doi.org/10.1136/annrheumdis-2017-211560

[2] Dhodapkar, K.M. (2019) Autoimmune Complications of Cancer Immunotherapy. Current Opinion in Immunology, 61, 54-59. https://doi.org/10.1016/j.coi.2019.08.004

[3] Jamal, S., Hudson, M., Fifi-Mah, A. and Ye, C. (2019) Immune Related Adverse Events Associated with Cancer Immunotherapy: A Review for the Practicing Rheumatologist. Journal of Rheumatology, 46. https://doi.org/10.3899/jrheum.190084

[4] Eun, Y., Kim, I.Y., Sun, J.M., Lee, J., Cha, H.S., Koh, E.M., Kim, H. and Lee, J. (2019) Risk Factors for Immune-Related Adverse Events Associated with Anti-PD-1 Pembrolizumab. Scientific Reports, 9, 14039. https://doi.org/10.1038/s41598-019-50574-6

[5] Liu, Y.H., Zang, X.Y., Wang, J.C., Huang, S.S. and Zhang, P. (2019) Diagnosis and Management of Immune Related Adverse Events (irAEs) in Cancer Immunotherapy. Biomedicine and Pharmacotherapy, 120, Article ID: 109437. https://doi.org/10.1016/j.biopha.2019.109437

[6] Japgal, A., Choudhary, G. and Chokr, J. (2019) Severe Arthritis and Tenosynovitis Caused by Immune Checkpoint Blocked Therapy with Pembrolizumab (Anti-PD-1 Antibody). Baylor University Medical Center Proceedings, 32, 419-421. https://doi.org/10.1080/08998280.2019.1588654

[7] Cappelli, L.C., Guttierrez, A.K., Bingham, C.O. and Shah, A.A. (2017) Rheumatic and Musculoskeletal Immune-Related Adverse Events Due to Immune Checkpoint Inhibitors: A Systematic Review of the Literature. Arthritis Care \& Research, 69, 1751-1763. https://doi.org/10.1002/acr.23177

[8] Kaur, A., Doberstein, T., Amberker, R.R., Garje, R., Field, E.H. and Singh, N. (2019) Immune-Related Adverse Events in Cancer Patients Treated with Immune Checkpoint Inhibitors: A Single-Center Experience. Medicine, 98, e17348. https://doi.org/10.1097/MD.0000000000017348

[9] Khan, Z., Hammer, C., Guardino, E., Scott Chandler, G.S. and Albert, M.L. (2019) Mechanisms of Immune-Related Adverse Events Associated with Immune Checkpoint Blockade: Using Germline Genetics to Develop a Personalized Approach. Genome Medicine, 11, 39. https://doi.org/10.1186/s13073-019-0652-8

[10] Trihn, S., Le, A., Gowani, S. and La-Beck, N.M. (2019) Management of ImmuneRelated Adverse Events Associated with Immune Checkpoint Inhibitor Therapy: A Minireview of Current Clinical Guidelines. Asia-Pacific Journal of Oncology Nursing, 6, 154-160. https://doi.org/10.4103/apjon.apjon_3_19

[11] Capelli, L.C., Naidoo, J., Bingham, C.O. and Shah, A.A. (2017) Inflammatory Arthritis Due to Immune Checkpoint Inhibitors Challenge in Diagnosis and Treatment. Immunotherapy, 9, 5-8. https://doi.org/10.2217/imt-2016-0117

[12] Kostine, M., Chiche, L., Lazaro, E., Halfon, P., Charpin, C., Arniaud, D., Retornaz, F., Blanco, P., Jourde-Chiche, N., Richez, C. and Stavris, C. (2017) Opportunistic Autoimmunity Secondary to Cancer Immunotherapy (OASI): An Emerging Challenge. La Revue de Médecine Interne, 38, 513-525. https://doi.org/10.1016/j.revmed.2017.01.004

[13] Boland, P., Heath, J. and Sandigursky, S. (2019) Immune Checkpoint Inhibitors and 
Vasculitis. Current Opinion in Rheumatology, 32, 53-56.

https://doi.org/10.1097/BOR.0000000000000672

[14] Richter, M.D., Crowson, C., Kottsade, L.A., Finnes, H.D., Marcovic, S.N. and Thanarajasingam, U. (2019) Rheumatic Syndromes Associated with Immune Checkpoint Inhibitors: A Single Cohort of Sixty-One Patients. Arthritis and Rheumatology, 71, 468-475. https://doi.org/10.1002/art.40745

[15] Lee, K.A., Kim, H.R. and Yoon, S.Y. (2019) Rheumatic Complication in Cancer Patients Treated with Immune Checkpoint Inhibitors. Korean Journal of Internal Medicine, 34, 1197-1209. https://doi.org/10.3904/kjim.2019.060 